\title{
MODELLING OF DIELECTRIC CAVITY STRUCTURES USING MULTIRESOLUTION TIME-DOMAIN ANALYSIS
}

\author{
rob robertson,* emmanouil tentzeris, michael krumpholz and linda p. b. katehi \\ Radiation Laboratory, Department of Electrical Engineering and Computer Science, University of Michigan, Ann Arbor, \\ MI 48109-2122, U.S.A.
}

\begin{abstract}
SUMMARY
Multiresolution time domain (MRTD) analysis is applied directly to Maxwell's equations to model inhomogeneous dielectric material. In our approach, scaling and wavelet functions are used as a complete basis for the method of moments. The MRTD scheme is used to analyze different types of resonant cavity structures with varying dielectric perturbations in one, two and three dimensions. The results presented here agree very well with those obtained by FDTD, FEM and integral equation methods. MRTD allows for considerable savings in memory and computation time in comparison to FDTD, while maintaining the same accuracy of the results. (C) 1998 John Wiley \& Sons, Ltd.
\end{abstract}

Int. J. Numer. Model., 11, 55-68 (1998)

\section{INTRODUCTION}

The method of moments ${ }^{1}$ is a mathematically correct approach for the discretization of integral and partial differential equations. This technique can be used to derive Yee's FDTD scheme using pulse functions for the expansion of the unknown fields. ${ }^{2,3}$ Since the method of moments allows for the use of a complete set of orthonormal basis functions, the choice of an appropriate set may lead to new discretization schemes. In the literature, ${ }^{4,5}$ the use of scaling and wavelet functions as a complete set of basis functions is called multiresolution analysis. The MRTD scheme is a fast-growing new method ${ }^{6-8}$ which incorporates the advantages of multiresolution analysis. It has been shown that the MRTD scheme has highly linear dispersion characteristics resulting in a high degree of accuracy in electromagnetic computations for discretizations close to the Nyquist limit. The MRTD scheme presented in this paper is extended for arbitrary resonant cavity structures, with varying dielectrics. Complete formulations for dielectrics in one, two and three dimensions have been developed, using both scaling and wavelet functions.

Battle-Lemarie ${ }^{9,10}$ scaling and wavelet functions are used to represent the electric and magnetic fields in several resonant cavity structures. The first is a cavity which is loaded with a quarterslice dielectric. In this case the $\epsilon$ coefficient varies only along one co-ordinate direction, which is referred to as a one-dimensional dielectric perturbation. Both the scaling function based MRTD (S-MRTD) and wavelet function based MRTD (Wy-MRTD) scheme achieved excellent correlation with FDTD results and analytic values for discretization close to the Nyquist limit. The second structure is a resonant cavity loaded with a dielectric slice along two co-ordinate directions, referred to as a two-dimensional dielectric perturbation. As with the previous structure, the SMRTD method achieved excellent correlation with FDTD results. The final analysis is performed with two different resonant cavities with a dielectric along three co-ordinate directions, referred to as a three-dimensional dielectric perturbation. In the first case, a cavity has a varying threedimensional dielectric perturbation in one corner of the structure. It is shown that S-MRTD gives excellent correspondence to FDTD in the frequency domain. The second case is a cavity with a varying three-dimensional dielectric perturbation in the centre of the bottom side. In this case SMRTD results are compared to results generated by FEM and integral equation (IE) methods. Once again excellent correspondence is achieved between S-MRTD, FEM and IE methods.

*Correspondence to: Rob Robertson, Department of Electrical Engineering and Computer Science, The University of Michigan, 3234 EECS Building, 1301 Beal Avenue, Ann Arbor, MI 48109-2122, U.S.A. 


\section{MODELLING INHOMOGENEOUS DIELECTRIC MATERIAL}

In the S-MRTD scheme, Battle-Lemarie scaling functions ${ }^{9,10}$ are used as a complete set of orthonormal basis functions. This scheme is derived in a homogeneous medium by representing the field components as a series of scaling and pulse functions in the space and time domains. ${ }^{11,12}$ For complete multiresolution analysis, first-order wavelet functions are added to produce the WyMRTD scheme. The Wy-MRTD scheme in a homogeneous medium is derived by representing the field components as a series of scaling and wavelet functions in the space domain and pulse functions in the time domain. The situation becomes more complicated for inhomogeneous dielectric media. Since Battle-Lemarie (BL) scaling functions are non-localized in space, they cannot account directly for localized boundary conditions. This Section describes how to discretize the appropriate constitutive relations for one-, two- and three-dimensional dielectric perturbations.

\subsection{General derivation}

To model the electromagnetic field inside an inhomogeneous dielectric material, Maxwell's first vector equation is separated in

$$
\nabla \times \mathbf{H}=\frac{\partial \mathbf{D}}{\partial t}
$$

and

$$
\mathbf{D}=\epsilon(\vec{r}, t) \mathbf{E}
$$

where D represents the electric flux vector and $\epsilon(\vec{r}, t)$ the space- and time-dependent permittivity tensor. These equations can be discretized using scaling and pulse functions in the space and time domains, respectively, as expansion factors in the method of moments. ${ }^{11,12}$ The use of nonlocalized basis functions cannot accommodate localized boundary conditions and cannot allow for a localized modelling of the material properties. To overcome this difficulty, the image principle is used to model perfect electric and magnetic boundary conditions. As for the description of material parameters, the constitutive relations are discretized accordingly so that the relationships between the electric/magnetic flux and the electric/magnetic field are given by matrix equations. In the following, the discretization of (2) using the method of moments is described.

In the principal co-ordinate system, the permittivity tensor $\boldsymbol{\epsilon}$ for symmetric media is given by

$$
\boldsymbol{\epsilon}(\vec{r}, t)=\left[\begin{array}{ccc}
\epsilon_{x}(\vec{r}, t) & 0 & 0 \\
0 & \epsilon_{y}(\vec{r}, t) & 0 \\
0 & 0 & \epsilon_{z}(\vec{r}, t)
\end{array}\right]
$$

In this case, (2) may be written in the form of three scalar Cartesian equations as

$$
\begin{aligned}
& D_{x}=\epsilon_{x}(\vec{r}, t) E_{x} \\
& D_{y}=\epsilon_{y}(\vec{r}, t) E_{y} \\
& D_{z}=\epsilon_{z}(\vec{r}, t) E_{z}
\end{aligned}
$$

For simplicity in the presentation, consider the discretization of (4), (5) and (6) for field expansions using only scaling functions in the space domain. Under this assumption the field expansions of the electric flux and electric field vectors are shown by the following set of equations:

$$
F_{x}(\vec{r}, t)=\sum_{k, l, m, n=-\infty}^{+\infty}{ }_{k} F_{l+1 / 2, m, n}^{\phi x} h_{k}(t) \phi_{l+1 / 2}(x) \phi_{m}(y) \phi_{n}(z)
$$




$$
\begin{aligned}
& \mathrm{F}_{y}(\vec{r}, t)=\sum_{k, l, m, n=-\infty}^{+\infty}{ }_{k} F_{l, m+1 / 2, n}^{\phi y} h_{k}(t) \phi_{l}(x) \phi_{m+1 / 2}(y) \phi_{n}(z) \\
& F_{z}(\vec{r}, t)=\sum_{k, l, m, n=-\infty}^{+\infty}{ }_{k} F_{l, m, n+1 / 2}^{\phi z} h_{k}(t) \phi_{l}(x) \phi_{m}(y) \phi_{n+1 / 2}(z)
\end{aligned}
$$

where $F_{\kappa}(\vec{r}, t)=\left[E_{\kappa}(\vec{r}, t), D_{\kappa}(\vec{r}, t)\right]$, with $\kappa=x, y, z$. The coefficients ${ }_{k} F_{l, m, n}^{\phi \kappa}$ represent the field expansion coefficients in terms of scaling functions. The indices $l, m, n$ and $k$ are the discrete space and time indices related to the space and time co-ordinates via $x=l \Delta x, y=m \Delta y, z=n \Delta z$ and $t=k \Delta t$, where $\Delta x, \Delta y, \Delta z$ and $\Delta t$ represent the space and time discretization intervals in the $x-, y-, z-$ and $t$-directions. The function $h_{m}(x)$ is defined as

$$
h_{m}(x)=h\left(\frac{x}{\Delta x}-m\right)
$$

where $h(x)$ is a rectangular pulse function given by

$$
h(x)=\left\{\begin{array}{cc}
1 & \text { for }|x|<1 / 2 \\
1 / 2 & \text { for }|x|=1 / 2 \\
0 & \text { for }|x|>1 / 2
\end{array}\right.
$$

The function $\phi_{m}(x)$ is defined as

$$
\phi_{m}(x)=\phi\left(\frac{x}{\Delta x}-m\right)
$$

where $\phi(x)$ represents the cubic spline Battle-Lemarie scaling functions. ${ }^{9,10}$

The field equations shown in (7) are inserted into (4), (5) and (6) and the equations are sampled using pulse functions with respect to time and scaling test functions with respect to space. Assuming

$$
\epsilon_{\kappa}(\vec{r}, t)=\epsilon_{\kappa}(x) \epsilon_{\kappa}(y) \epsilon_{\kappa}(z) \epsilon_{\kappa}(t)
$$

and sampling (4) with $\phi_{l+1 / 2}(x) \phi_{m}(y) \phi_{n}(z) h_{k}(t)$ yields

$$
{ }_{k} D_{l+1 / 2, m, n}^{\phi x}=\sum_{k^{\prime}, l^{\prime}, m^{\prime}, n^{\prime}=-\infty}^{+\infty} \epsilon_{(x) l+1 / 2, l+1 / 2^{\prime}}^{\phi x} \epsilon_{(y) m, m^{\prime}}^{\phi x} \epsilon_{(z) n, n^{\prime}}^{\phi x} \epsilon_{(t), k, k^{\prime}}^{x} k^{\prime} E_{l^{\prime}+1 / 2, m^{\prime}, n^{\prime}}^{\phi z}
$$

where $\epsilon_{(\kappa) m, m^{\prime}}^{\phi x}$ and $\boldsymbol{\epsilon}_{(t) k, k^{\prime}}^{x}$ are integrals given by

$$
\epsilon_{(\kappa) m, m^{\prime}}^{\phi x}=\frac{1}{\Delta \kappa} \int_{-\infty}^{+\infty} \phi_{m}(\kappa) \epsilon_{x}(\kappa) \phi_{m^{\prime}}(\kappa) \mathrm{d} \kappa
$$

and

$$
\epsilon_{(t) k, k^{\prime}}^{x}=\frac{1}{\Delta t} \int_{-\infty}^{+\infty} h_{k}(t) \epsilon_{x}(t) h_{k^{\prime}}(t) \mathrm{d} t
$$

In subsequent Sections, the derivation of specific expressions for the dielectrics in one, two and three dimensions are presented. 


\subsection{Analysis of resonant cavities with one-dimensional dielectrics}

In this Section the equations describing a dielectric perturbation that varies only along one direction are derived. Consider, for example, the structure shown in Figure 1. This structure has a dielectric constant which varies only along the $y$-axis and is homogeneous along the $x$ - and $z$ directions, with no time dependence. Therefore, the non-zero elements of the permittivity tensor are as follows:

$$
\begin{aligned}
& \epsilon_{x}(\vec{r}, t)=\epsilon_{x}(y) \\
& \epsilon_{y}(\vec{r}, t)=\epsilon_{y}(y) \\
& \epsilon_{x}(\vec{r}, t)=\epsilon_{z}(y)
\end{aligned}
$$

Applying (15), (16) and (17) to (4), (5) and (6), and using the field expansions in (7), yields:

$$
\begin{gathered}
{ }_{k} D_{l+1 / 2, m, n}^{\phi x}=\sum_{m^{\prime}=-\infty}^{+\infty} \epsilon_{(y) m, m^{\prime} k^{\prime}}^{\phi x} E_{l^{\prime}+1 / 2, m^{\prime}, n^{\prime}}^{\phi x} \\
{ }_{k} D_{l, m+1 / 2, n}^{\phi y}=\sum_{m^{\prime}=-\infty}^{+\infty} \epsilon_{(y) m+1 / 2, m^{\prime}+1 / 2{ }^{\prime}{ }^{\prime}}^{\phi y} E_{l^{\prime}, m^{\prime}+1 / 2, n^{\prime}}^{\phi y} \\
{ }_{k} D_{l, m, n+1 / 2}^{\phi z}=\sum_{m^{\prime}=-\infty}^{+\infty} \epsilon_{(y) m, m^{\prime} k^{\prime}}^{\phi z} E_{l^{\prime}, m^{\prime}, n^{\prime}+1 / 2}^{\phi z}
\end{gathered}
$$

where the integrals $\boldsymbol{\epsilon}_{(y) m, m^{\prime}}^{\phi x}, \boldsymbol{\epsilon}_{(y) m+1 / 2, m^{\prime}+1 / 2}^{\phi y}$ and $\boldsymbol{\epsilon}_{(y) m, m^{\prime}}^{\phi z}$ are given by:

$$
\begin{aligned}
\boldsymbol{\epsilon}_{(y) m, m^{\prime}}^{\phi x} & =\frac{1}{\Delta y} \int_{-\infty}^{+\infty} \phi_{m}(y) \varepsilon_{x}(y) \phi_{m^{\prime}}(y) \mathrm{d} y \\
\boldsymbol{\epsilon}_{(y) m+1 / 2, m^{\prime}+1 / 2}^{\phi y} & =\frac{1}{\Delta y} \int_{-\infty}^{+\infty} \phi_{m+1 / 2}(y) \epsilon_{y}(y) \phi_{m^{\prime}+1 / 2}(y) \mathrm{d} y \\
\boldsymbol{\epsilon}_{(y) m, m^{\prime}}^{\phi z} & =\frac{1}{\Delta y} \int_{-\infty}^{+\infty} \phi_{m}(y) \epsilon_{z}(y) \phi_{m^{\prime}}(y) \mathrm{d} y
\end{aligned}
$$

Note that although the limits of integration are infinite the Battle-Lemarie scaling functions exponentially decay to zero after $\pm 6 \Delta l$, which significantly truncates the limits of the integration.

For the evaluation of the integrals, (21)-(23), a simple representation of the scaling function in terms of cubic spline functions is used. ${ }^{12}$ The structure shown in Figure 1 is used to illustrate an evaluation of the integrals. This structure is a resonant cavity that is one-quarter filled with a dielectric material. The cavity has the dimensions $1 \mathrm{~cm} \times 2 \mathrm{~cm} \times 1.5 \mathrm{~cm}$, and the dielectric material has a relative dielectric constant equal to 10 . A discretization of $2 \times 6 \times 3$ is applied for the method described below. The electric field components tangential to the dielectric interface,

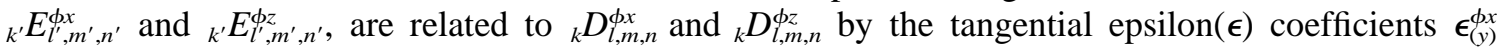

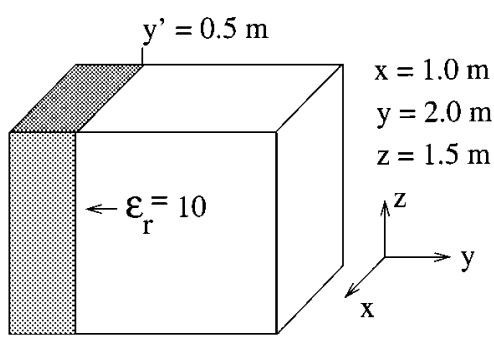

Figure 1. Cavity resonator one-quarter filled with dielectric 


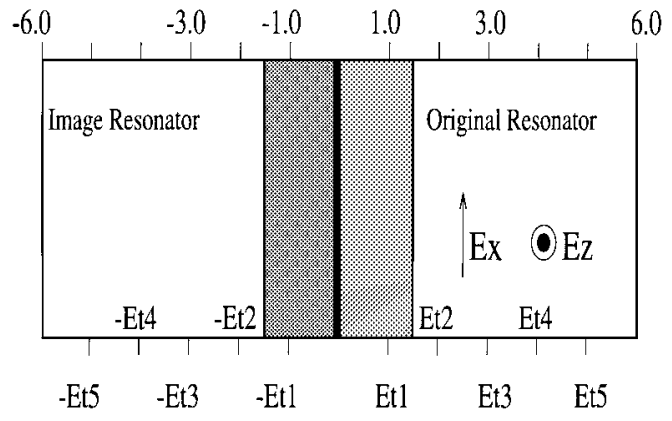

Figure 2. Tangential epsilon components

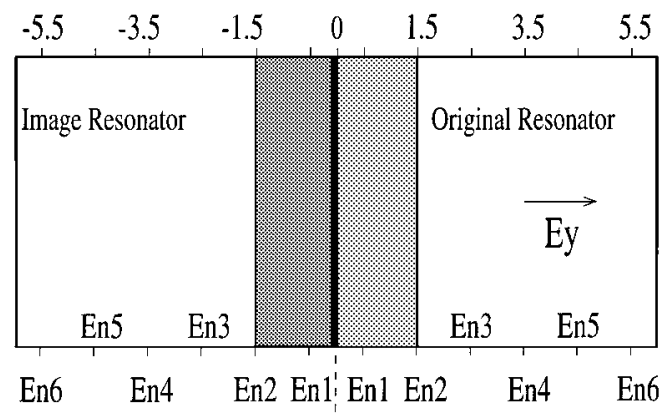

Figure 3. Normal epsilon components

and $\epsilon_{(y)}^{\phi z}$, respectively. Additionally, the electric field component normal to the dielectric interface $k^{\prime} E_{l^{\prime}, m^{\prime}, n^{\prime}}^{\phi y}$ is related to ${ }_{k^{\prime}} D_{l^{\prime}, m^{\prime}, n^{\prime}}^{\phi y}$ by the normal $(\epsilon)$ coefficient $\epsilon_{(y)}^{\phi y}$. To model the structure in Figure 1 the image principle is applied, thus replacing the structure in Figure 1 by the structures shown in Figures 2 and 3, respectively. The tangential $\epsilon$ coefficients now relate ten ${ }_{k^{\prime}} E_{l^{\prime}, m^{\prime}, n^{\prime}}^{x}$ components to ten ${ }_{k} D_{l, m, n}^{\phi x}$ components through a $10 \times 10$ matrix. The image principle applies odd symmetry of the tangential electric fields. Thus the five tangential electric field components in the image resonator are linearly dependent on the five tangential electric field components in the original resonator. This allows the elimination of the field components in the image resonator, reducing the $10 \times 10$ matrix to a $5 \times 5$ matrix, which is used in (8). Similarly, the $12 \times 12$ matrix of the normal $\epsilon$ coefficient is reduced to a $6 \times 6$ matrix using even symmetry for the normal electric field component. Note that a general description of $\epsilon$ coefficients in (9) allows for an arbitrary positioning of the dielectric interface.

The MRTD method for the structure in Figure 1 at a discretization of $2 \times 6 \times 3$ proved to be the closest approximation to analytic values. This is due to to the fact that a discretization of $2 \times 4 \times 3$ is nearly at the Nyquist criterion for the variation of $\epsilon$ in the $y$-direction (one sampling point in the dielectric material).

MRTD results are compared to analytic values and the results obtained by Yee's FDTD scheme in Table I. The same value for the time discretization interval $\Delta t=0.9 \times 10^{-9} \mathrm{~s}$ is used for both schemes. This time step interval is chosen to maximize the linear properties of the MRTD dispersion relation. ${ }^{12}$ Both cases run for 35,000 time steps. For the analysis using Yee's FDTD scheme, a mesh with $\Delta x=\Delta y=\Delta z=0 \cdot 1 \mathrm{~cm}$ is used, resulting in a total number of 3000 grid points. In the $2 \times 6 \times 3$ analysis, a total number of 36 grid points is used, resulting in a memory improvement by a factor of 83 for MRTD. Additionally an improvement by a factor of 10 in computation time is found for the MRTD method.

Table I. Resonant frequency data for a cavity one-quarter filled with dielectric material

\begin{tabular}{lrrr}
\hline Analytic, GHz & $6 \cdot 6990$ & $8 \cdot 7805$ & $9 \cdot 3007$ \\
FDTD $(10 \times 20 \times 15)$ & 6.686 & $8 \cdot 770$ & $9 \cdot 270$ \\
FDTD relative error \% & -0.194 & -0.119 & -0.330 \\
S-MRTD $(2 \times 6 \times 3)$ & 6.707 & $8 \cdot 800$ & 9.343 \\
MRTD relative error \% & $0 \cdot 119$ & 0.222 & 0.435 \\
\hline
\end{tabular}


Due to the properties of multiresolution analysis, MRTD has a unique ability to calculate field distributions. While a method such as FDTD results in only one field value per discretization cell, MRTD scaling functions imply field variations within the discretization cell. Deriving MRTD and FDTD using the method of moments, the field components have to be interpreted as field expansion coefficients. From the different field expressions, it is clear that the field expansion coefficients of the FDTD scheme represent the total field value at a specific point, while the field expansion coefficients of the MRTD scheme represent a fraction of the total field. To calculate the total field at a space point, the field expansions are sampled with delta test functions in the space and time domains. For example, the total electric field $E_{y}\left(x_{o}, y_{o}, z_{o}, t_{o}\right)$ with $(k-1 / 2) \Delta t<t_{o}<(k+1 / 2) \Delta t$ is calculated by

$$
\begin{aligned}
E_{x}\left(x_{o}, y_{o}, z_{o}, t_{o}\right) & =\iiint \int E_{y}(x, y, z, t) \delta\left(x-x_{o}\right) \delta\left(y-y_{o}\right) \delta\left(z-z_{o}\right) \delta\left(t-t_{o}\right) \mathrm{d} x \mathrm{~d} y \mathrm{~d} z \mathrm{~d} t \\
& =\sum_{l^{\prime}, m^{\prime}, n^{\prime}=-\infty}^{\infty}{ }_{k} E_{l^{\prime}, m^{\prime},+1 / 2, n^{\prime}} \phi_{l^{\prime}}\left(x_{o}\right) \phi_{m^{\prime}+1 / 2}\left(y_{o}\right) \phi_{n^{\prime}}\left(z_{o}\right)
\end{aligned}
$$

Practically, the above summation is truncated to very few terms (6-8 per index), due to the exponentially decaying support of the Battle-Lemarie scaling function.

Figures 4 and 5 show plots of $E^{\phi y}$ calculated using S-MRTD with a discretization of $2 \times 8 \times 3$ for the quarter-slice dielectric. Figure 4 shows the amplitudes of the scaling functions calculated by MRTD. Figure 5 shows field distributions using seven intermediate points along each of the co-ordinate axes. Figure 6 shows a field plot calculated by FDTD with a discretization of $20 \times 40 \times 30$. It should be noted that execution time for the interpolation was extremely low.

As mentioned above, for multiresolution analysis, wavelets must be added to discretize Maxwell's equations in the space domain. Therefore, consider the discretization of (4)-(6) for field expansions with scaling and wavelet functions in the space domain. The expansions of the field components are now a two-fold expansion in scaling and wavelet functions with respect to the $y$-axis as shown below:

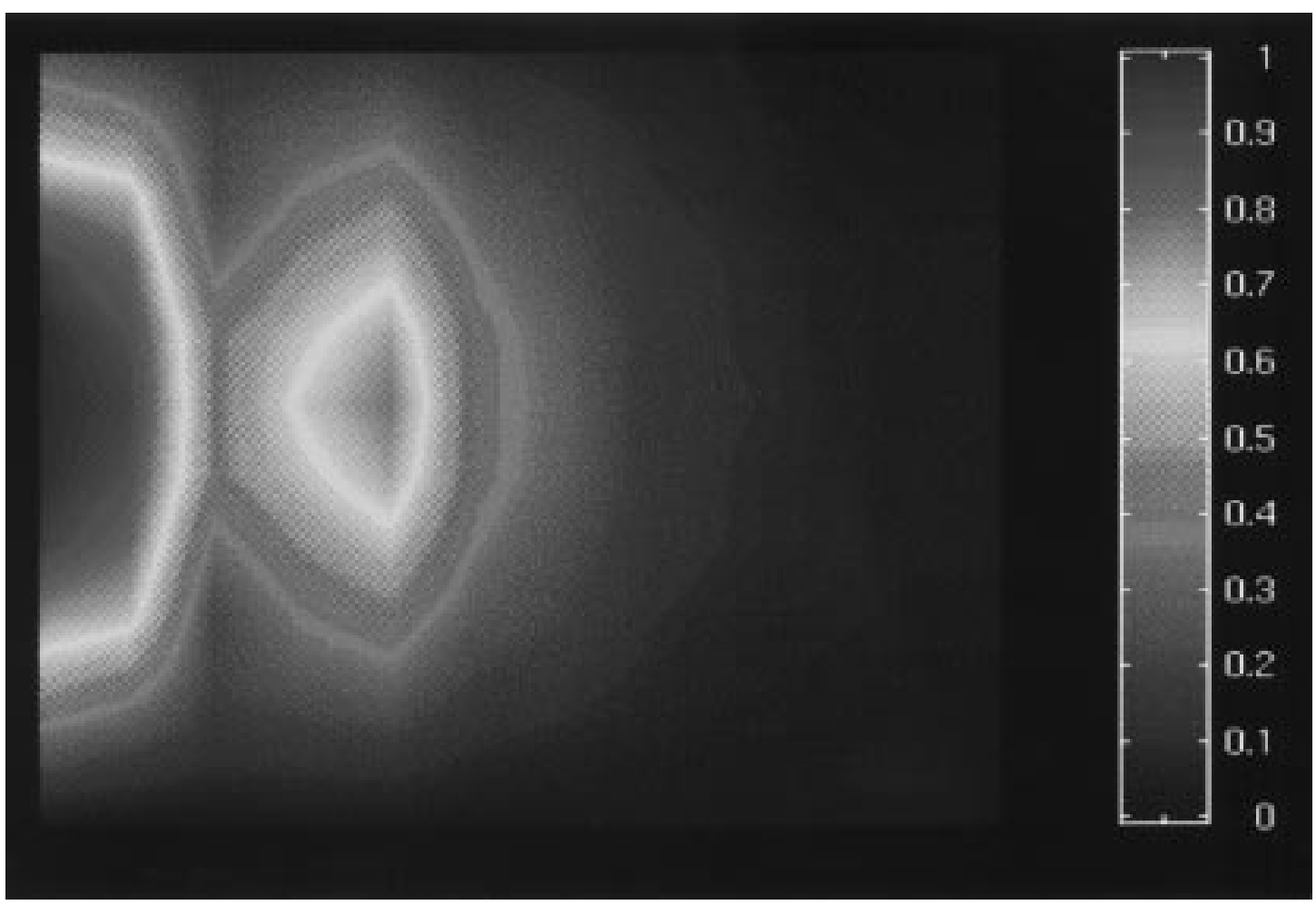

Figure 4. S-MRTD field pattern for quarter dielectric, not interpolated 


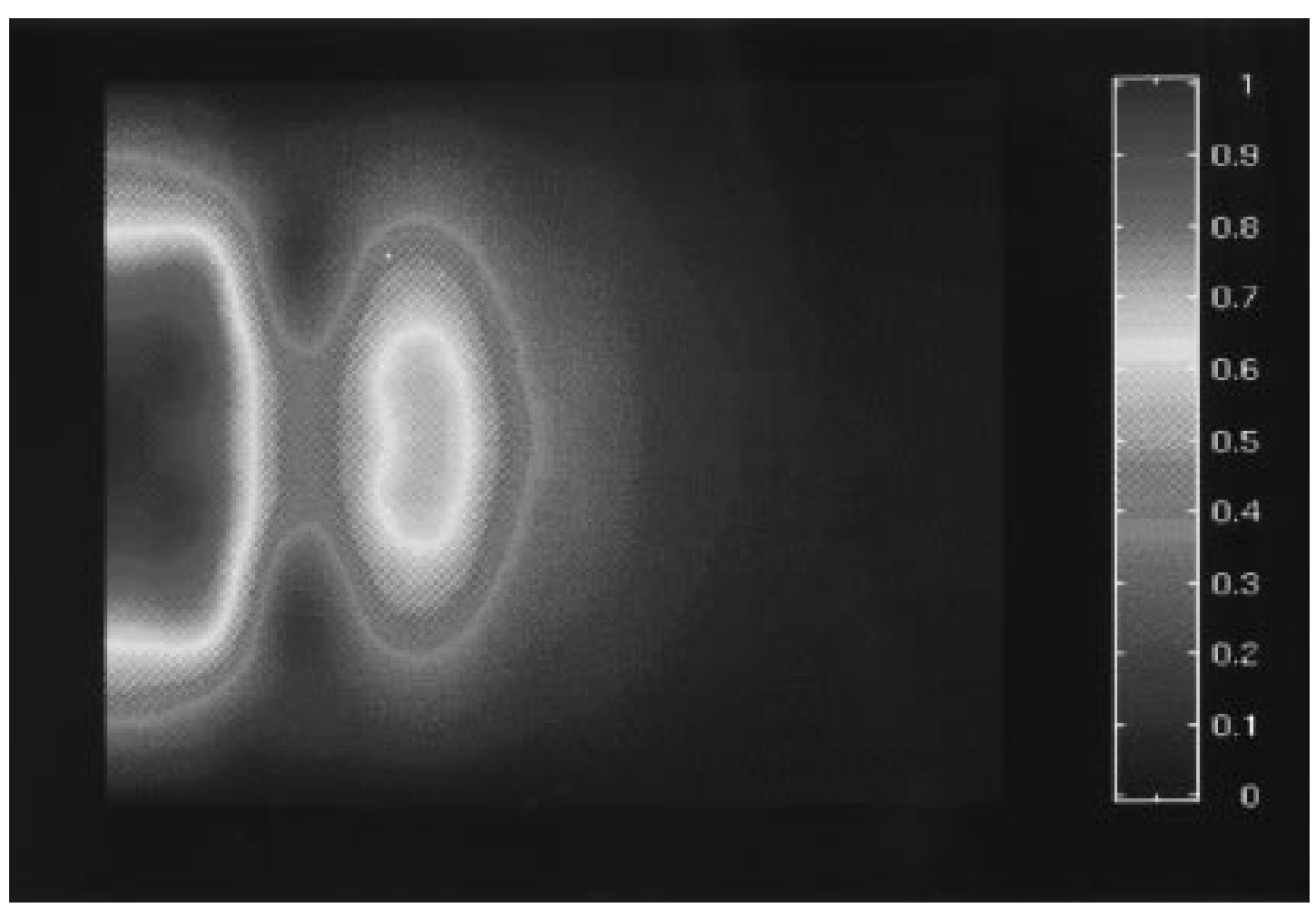

Figure 5. S-MRTD field pattern for quarter dielectric, interpolated

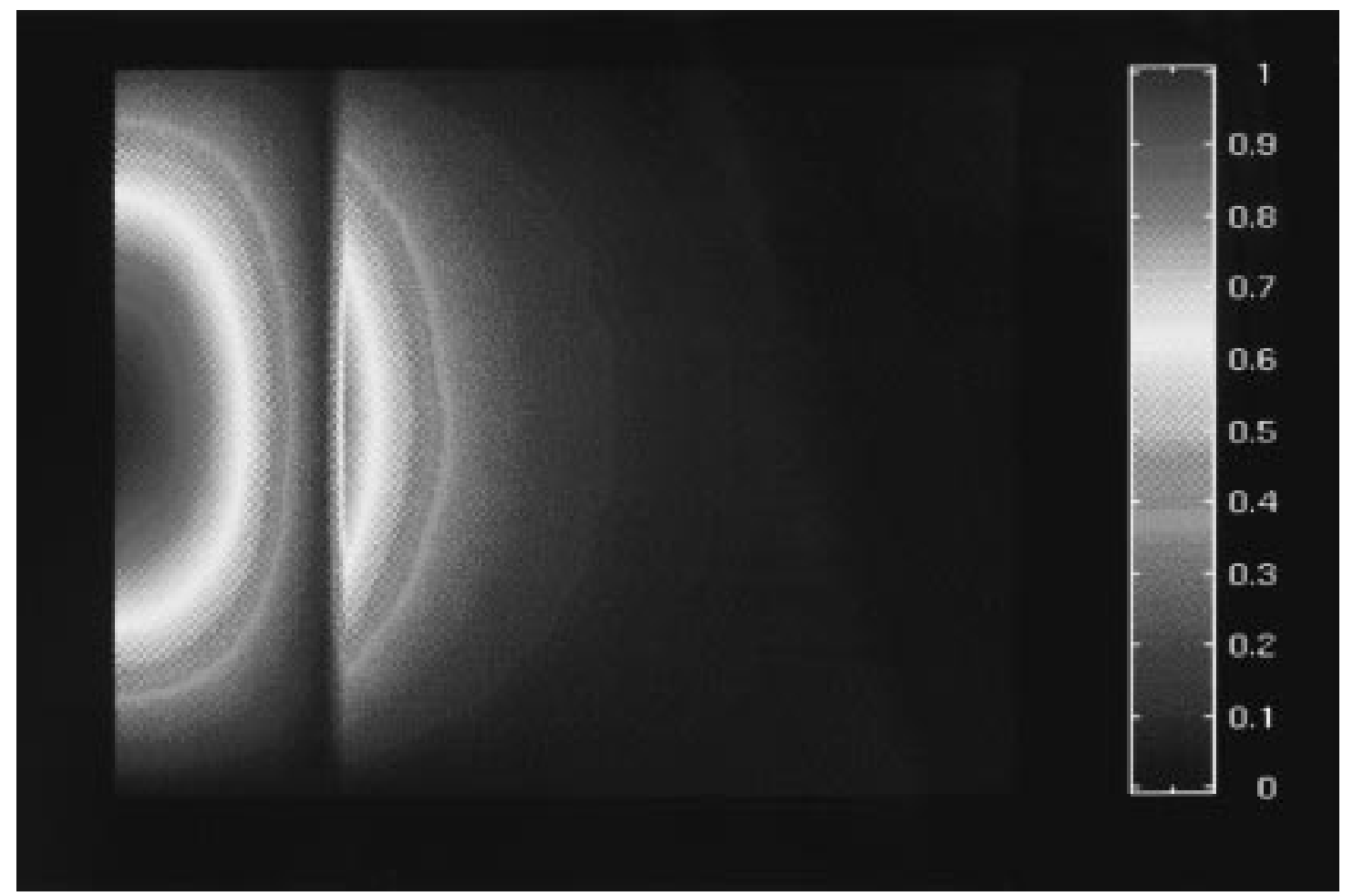

Figure 6. FDTD field pattern for quarter dielectric

$$
\begin{aligned}
& F_{x}(\vec{r}, t)=\sum_{k, l, m, n=-\infty}^{+\infty}\left({ }_{k} F_{l+1 / 2, m, n}^{\phi x} \phi_{m}(y)+{ }_{k} F_{l+1 / 2, m+1 / 2, n}^{\psi x} \psi_{m+1 / 2}(y)\right) h_{k}(t) \phi_{l+1 / 2}(x) \phi_{n}(z) \\
& F_{y}(\vec{r}, t)=\sum_{k, l, m, n=-\infty}^{+\infty}\left({ }_{k} F_{l, m+1 / 2, n}^{\phi y} \phi_{m+1 / 2}(y)+{ }_{k} F_{l, m, n}^{\psi y} \psi_{m}(y)\right) h_{k}(t) \phi_{l}(x) \phi_{n}(z)
\end{aligned}
$$




$$
F_{z}(\vec{r}, t)=\sum_{k, l, m, n=-\infty}^{+\infty}\left({ }_{k} F_{l, m, n+1 / 2}^{\phi z} \phi_{m}(y)+{ }_{k} F_{l, m+1 / 2, n+1 / 2}^{\psi z} \psi_{m+1 / 2}(y)\right) h_{k}(t) \phi_{l}(x) \phi_{n+1 / 2}(z)
$$

where $F_{\kappa}(\vec{r}, t)=\left(E_{\kappa}(\vec{r}, t), D_{\kappa}(\vec{r}, t)\right)$ with $\kappa=x, y, z$. Inserting (15), (16) and (17) into (4), (5) and (6) while using the expansions given in (25) and the procedure discussed above yields:

$$
\begin{aligned}
& { }_{k} D_{l+1 / 2, m, n}^{\phi x}=\sum_{m^{\prime}=-\infty}^{+\infty}\left(\epsilon_{(y) m, m^{\prime} k}^{\phi x} E_{l+1 / 2, m^{\prime}, n}^{\phi x}+\epsilon_{(y) m, m^{\prime}+1 / 2{ }_{k}}^{I x} E_{l+1 / 2, m^{\prime}+1 / 2, n}^{\psi x}\right) \\
& { }_{k} D_{l+1 / 2, m+1 / 2, n}^{\psi x}=\sum_{m^{\prime}=-\infty}^{+\infty}\left(\epsilon_{(y) m^{\prime}, m+1 / 2 k}^{I x} E_{l+1 / 2, m^{\prime}, n}^{\phi x}+\epsilon_{(y) m+1 / 2, m^{\prime}+1 / 2 k}^{\psi x} E_{l+1 / 2, m^{\prime}+1 / 2, n}^{\psi x}\right) \\
& { }_{k} D_{l, m+1 / 2, n}^{\phi y}=\sum_{m^{\prime}=-\infty}^{+\infty}\left(\epsilon_{(y) m+1 / 2, m^{\prime}+1 / 2 k}^{\phi y} E_{l, m^{\prime}+1 / 2, n}^{\phi y}+\epsilon_{(y) m+1 / 2, m^{\prime}}^{I y} E_{l, m^{\prime}, n}^{\psi y}\right) \\
& { }_{k} D_{l, m, n}^{\psi y}=\sum_{m^{\prime}=-\infty}^{+\infty}\left(\epsilon_{(y) m^{\prime}+1 / 2, m k}^{I y} E_{l, m^{\prime}+1 / 2, n}^{\phi y}+\epsilon_{(y) m, m^{\prime} k}^{\psi y} E_{l, m^{\prime}, n}^{\psi y}\right) \\
& { }_{k} D_{l, m, n+1 / 2}^{\phi z}=\sum_{m^{\prime}=-\infty}^{+\infty}\left(\epsilon_{(y) m, m^{\prime}}^{\phi z} E_{l, m^{\prime}, n+1 / 2}^{\phi z}+\epsilon_{(y) m^{\prime}, m+1 / 2 k}^{I z} E_{l, m^{\prime}+1 / 2, n+1 / 2}^{z^{\prime}}\right) \\
& { }_{k} D_{l, m+1 / 2, n+1 / 2}^{\psi z}=\sum_{m^{\prime}=-\infty}^{+\infty}\left(\epsilon_{(y) m+1 / 2, m^{\prime}{ }_{k}}^{I z} E_{l, m^{\prime}, n+1 / 2}^{\phi z}+\epsilon_{(y) m+1 / 2, m^{\prime}+1 / 2 k}^{\psi z} E_{l, m^{\prime}+1 / 2, n+1 / 2}^{\psi z}\right)
\end{aligned}
$$

The new generalized integral identities are:

$$
\boldsymbol{\epsilon}_{(y) m, m^{\prime}}^{\psi \kappa}=\frac{1}{\Delta y} \int_{-\infty}^{+\infty} \psi_{m}(y) \epsilon_{\kappa}(y) \psi_{m^{\prime}}(y) \mathrm{d} y
$$

and

$$
\boldsymbol{\epsilon}_{(y) m, m^{\prime}}^{I \kappa}=\frac{1}{\Delta y} \int_{-\infty}^{+\infty} \phi_{m}(y) \boldsymbol{\epsilon}_{\kappa}(y) \psi_{m^{\prime}}(y) \mathrm{d} y
$$

The procedure for evaluating the integrals is similar to the procedure discussed for equations (21)-(23).

Data for the structure shown in Figure 1 are presented in Table II, with four and six wavelets added along the $y$-direction. As can be seen from Table II, adding wavelets increases the resonant frequency resolution.

\subsection{Analysis of resonant cavity with $2 D$ dielectric}

This Section shows the derivation of the equations describing a dielectric perturbation that varies along two co-ordinate directions. Consider the structure shown in Figure 7, where the

Table II. Resonant frequency data for a cavity one-quarter filled with dielectric material with the application of wavelets along the $y$-direction

\begin{tabular}{llll}
\hline Analytic, GHz & 6.699 & $8 \cdot 7805$ & $9 \cdot 300$ \\
Wy-MRTD (6 wavelets) & 6.703 & 8.7825 & $9 \cdot 315$ \\
Wy-MRTD relative error \% & 0.0596 & 0.0227 & $0 \cdot 1613$ \\
Wy-MRTD (4 wavelets) & 6.705 & 8.7850 & $9 \cdot 325$ \\
Wy-MRTD relative error, \% & 0.0894 & 0.05122 & 0.268 \\
\hline
\end{tabular}




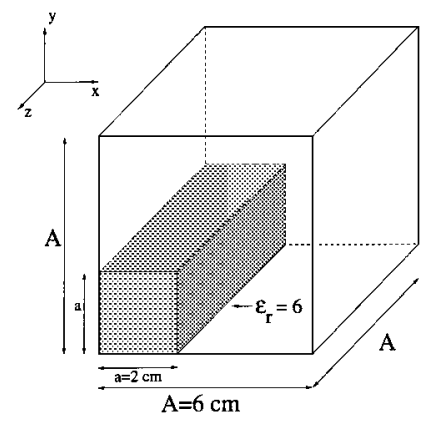

Figure 7. 2D dielectric structure

dielectric varies along the $x$ - and $y$-directions and is constant in the $z$-direction. In this structure, the non-zero elements of the permittivity tensor are as follows:

$$
\begin{aligned}
& \epsilon_{x}(\vec{r}, t)=\epsilon_{x}(x, y) \\
& \epsilon_{y}(\vec{r}, t)=\epsilon_{y}(x, y) \\
& \epsilon_{z}(\vec{r}, t)=\epsilon_{z}(x, y)
\end{aligned}
$$

Following the procedure above, (34), (35) and (36) are applied to (4), (5) and (6). Then, using the field expansions given by (7) yields

$$
\begin{aligned}
& { }_{k} D_{l+1 / 2, m, n}^{\phi x}=\sum_{l^{\prime}, m^{\prime}=-\infty}^{+\infty} \epsilon_{(x, y) l+1 / 2, l^{\prime}+1 / 2, m, m^{\prime}}^{\phi x} k^{\prime} E_{l^{\prime}+1 / 2, m^{\prime}, n^{\prime}}^{\phi x} \\
& { }_{k} D_{l, m+1 / 2, n}^{\phi y}=\sum_{l^{\prime}, m^{\prime}=-\infty}^{+\infty} \epsilon_{(x, y), l^{\prime}, m+1 / 2, m^{\prime}+1 / 2}^{\phi y} k^{\prime} E_{l^{\prime}, m^{\prime}+1 / 2, n^{\prime}}^{\phi y} \\
& { }_{k} D_{l, m, n+1 / 2}^{\phi z}=\sum_{n^{\prime}=-\infty}^{+\infty} \epsilon_{(x, y) l, l^{\prime}, m, m^{\prime} k^{\prime}}^{\phi z} E_{l^{\prime}, m^{\prime}, n^{\prime}+1 / 2}^{\phi z}
\end{aligned}
$$

where the integrals $\epsilon_{(x, y) l+1 / 2, l^{\prime}+1 / 2, m, m^{\prime}}^{\phi x}, \epsilon_{(x, y) l, l^{\prime}, m+1 / 2, m^{\prime}+1 / 2}^{\phi y}$ and $\epsilon_{(z) l, l^{\prime}, m, m^{\prime}}^{\phi z}$ are given by:

$$
\begin{aligned}
\epsilon_{(x, y) l+1 / 2, l^{\prime}+1 / 2, m, m^{\prime}}^{\phi x} & =\frac{1}{\Delta x \Delta y} \int_{-\infty}^{+\infty} \int_{-\infty}^{+\infty} \epsilon_{x}(x, y) \phi_{l+1 / 2}(x) \phi_{l^{\prime}+1 / 2}(x) \phi_{m}(y) \phi_{m^{\prime}}(y) \mathrm{d} x \mathrm{~d} y \\
\epsilon_{(x, y) l l^{\prime}, m+1 / 2, m^{\prime}+1 / 2}^{\phi y} & =\frac{1}{\Delta x \Delta y} \int_{-\infty}^{+\infty} \int_{-\infty}^{+\infty} \epsilon_{y}(x, y) \phi_{l}(x) \phi_{l^{\prime}}(x) \phi_{m+1 / 2}(y) \phi_{m^{\prime}+1 / 2}(y) \mathrm{d} x \mathrm{~d} y \\
\epsilon_{(x, y) l, l^{\prime}, m, m^{\prime}}^{\phi z} & =\frac{1}{\Delta x \Delta y} \int_{-\infty}^{+\infty} \int_{-\infty}^{+\infty} \epsilon_{z}(x, y) \phi_{l}(x) \phi_{l^{\prime}}(x) \phi_{m}(y) \phi_{m^{\prime}}(y) \mathrm{d} x \mathrm{~d} y
\end{aligned}
$$

The evaluation of (40) and (41) is similar to the procedure described for one-dimensional dielectric perturbations. This procedure is illustrated by applying a discretization of $6 \times 6 \times 6$ to the structure in Figure 7. This structure has the dimensions $6 \mathrm{~cm} \times 6 \mathrm{~cm} \times 6 \mathrm{~cm}$, with a onethird dielectric slice along the $x$ - and $y$-directions. The dielectric has a relative permittivity equal to 6. Here, the electric field components ${ }_{k^{\prime}} E_{l^{\prime}, m^{\prime}, n^{\prime}}^{x^{\prime}}$ and ${ }_{k^{\prime}} E_{l^{\prime}, m^{\prime}, n^{\prime}}^{\phi y}$ are related to ${ }_{k} D_{l, m, n}^{\phi x}$ and ${ }_{k} D_{l, m, n}^{\phi y}$ by a $6 \times 6 \times 6 \times 6$ matrix described by $\epsilon_{(x, y)}^{\phi x}$ and $\epsilon_{(x, y)}^{\phi y}$, respectively. To evaluate the integrals, the image theory is applied, thus replacing Figure 7 by a new structure made by imaging Figure 7 about the $x$ and $y$ axes. Now the electric field components ${ }_{k^{\prime}} E_{l^{\prime}, m^{\prime}, n^{\prime}}^{x}$ and ${ }_{k^{\prime}} E_{l^{\prime}, m^{\prime}, n^{\prime}}^{\phi y}$ are related to ${ }_{k} D_{l, m, n}^{\phi x}$ and ${ }_{k} D_{l, m, n}^{\phi y}$ by a $6 \times 6 \times 12 \times 12$ matrix. Each of the $\epsilon_{(x, y)}^{\phi x}$ and $\epsilon_{(x, y)}^{\phi y}$ matrix components is calculated by applying (40) and (41) over the new imaged structure. The epsilon components in each of the three resonators are linearly dependent on the components in the original 
Table III. Resonant frequency data for a cavity one-quarter filled with dielectric material

\begin{tabular}{ll}
\hline FDTD $(40 \times 40 \times 40), \mathrm{GHz}$ & 1.9640 \\
S-MRTD $(6 \times 6 \times 6)$ & 1.9480 \\
FDTD/MRTD relative error, $\%$ & 0.814 \\
S-MRTD $(8 \times 8 \times 8)$ & 1.9610 \\
FDTD/MRTD relative error, $\%$ & $0 \cdot 152$ \\
S-MRTD $(10 \times 10 \times 10)$ & 1.9610 \\
MRTD relative error, $\%$ & $0 \cdot 152$ \\
\hline
\end{tabular}

resonator. Therefore, the image theory is applied again, reducing the $6 \times 6 \times 12 \times 12$ matrix to a $6 \times 6 \times 6 \times 6$ matrix. Note that this can be repeated for any discretization along the $x$-, $y$ - or $z$-directions.

S-MRTD results for the structure in Figure 7 are shown in Table III. A time discretization interval of $\Delta t=1.0 \times 10^{-12} \mathrm{~s}$ and 80,000 time steps is used for FDTD, which has a discretization of $40 \times 40 \times 40$. The time discretization interval for S-MRTD $6 \times 6 \times 6$, S-MRTD $8 \times 8 \times 8$ and S-MRTD $10 \times 10 \times 10$ is $\Delta t=3 \cdot 0 \times 10^{-12} \mathrm{~s}$. In all three cases 60,000 time steps were used to generate frequency data. The S-MRTD discretization of $8 \times 8 \times 8$ provided excellent correlation with the FDTD method, resulting in a memory saving of a factor of 125 . Additionally execution time for the S-MRTD method was found to be significantly lower. Figures 8 , 9 and 10 show field distributions for S-MRTD and FDTD. Figure 8 is a plot of the $E^{\phi y}$ field for the noninterpolated S-MRTD method with a discretization of $8 \times 8 \times 8$. Figure 9 is an interpolated plot of the $E^{\phi y}$ distribution with seven intermediate points, resulting in a total discretization of $56 \times 56 \times 56$. The FDTD field plot shown in Figure 10 has a discretization of $40 \times 40 \times 40$. Note the high degree of correlation between the S-MRTD and FDTD field plots.

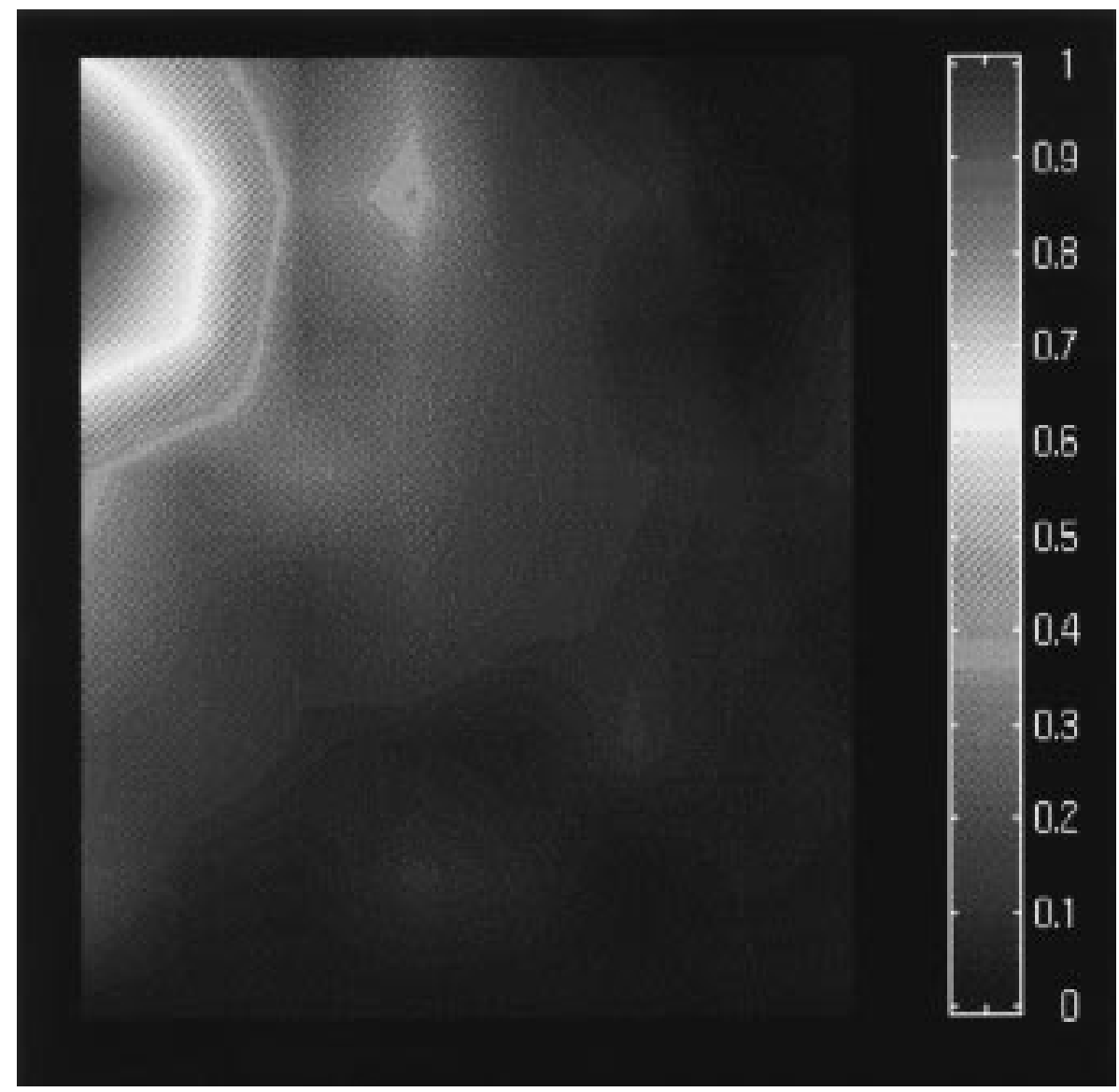

Figure 8. MRTD field pattern for 2D dielectric structure 


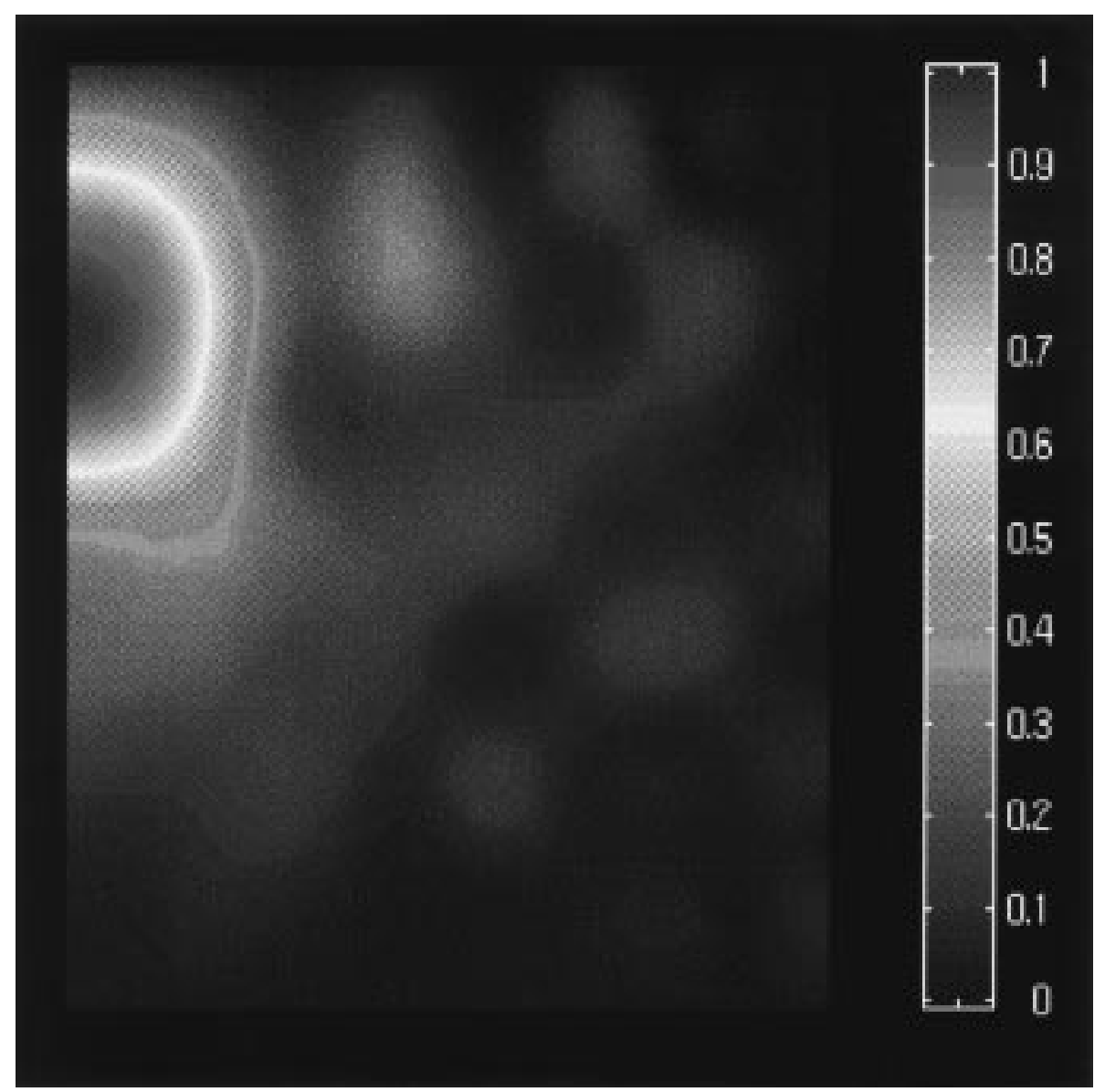

Figure 9. MRTD interpolated field pattern for 2D dielectric structure

\subsection{Analysis of resonant cavity with $3 D$ dielectric}

The equations describing a dielectric perturbation that varies along all three co-ordinate directions is described in this Section. Consider the structures shown in Figures 11 and 12, where the dielectric varies along the $x$-, $y$ - and $z$-directions. In this structure, the non-zero elements of the permittivity tensor are as follows:

$$
\begin{aligned}
& \epsilon_{x}(\vec{r}, t)=\epsilon_{x}(x, y, z) \\
& \epsilon_{y}(\vec{r}, t)=\epsilon_{y}(x, y, z) \\
& \epsilon_{z}(\vec{r}, t)=\epsilon_{z}(x, y, z)
\end{aligned}
$$

Applying a similar procedure to (43), (44) and (45), the following results for ${ }_{k} D_{l+1 / 2, m, n}^{\phi x}$ are received:

$$
{ }_{k} D_{l+1 / 2, m, n}^{\phi x}=\sum_{l^{\prime}, m^{\prime}, n^{\prime}=-\infty}^{+\infty} \epsilon_{(x, y, z) l+1 / 2, l^{\prime} / 1 / 2, m, m^{\prime}, n, n^{\prime}}^{\phi x} k^{\prime} E_{l^{\prime}+1 / 2, m^{\prime}, n^{\prime}}^{\phi x}
$$

The $\epsilon_{(x, y, z) l+1 / 2, l^{\prime}+1 / 2, m, m^{\prime}, n, n^{\prime}}^{\phi x}$ integral is given by

$$
\begin{aligned}
\epsilon_{(x, y, z) l+1 / 2, l^{\prime}+1 / 2, m, m^{\prime}, n, n^{\prime}}^{\phi x} & =\frac{1}{\Delta x \Delta y \Delta z} \int_{-\infty}^{+\infty} \int_{-\infty}^{+\infty} \int_{-\infty}^{+\infty} \epsilon_{x}(x, y, z) \phi_{l+1 / 2}(x) \phi_{l^{\prime}+1 / 2}(x) \\
& \times \phi_{m}(y) \phi_{m^{\prime}}(y) \phi_{n}(z) \phi_{n^{\prime}}(z) \mathrm{d} x \mathrm{~d} y \mathrm{~d} z
\end{aligned}
$$

The first structure considered is a resonant cavity $6 \mathrm{~cm}$ on a side with a varying dielectric of 


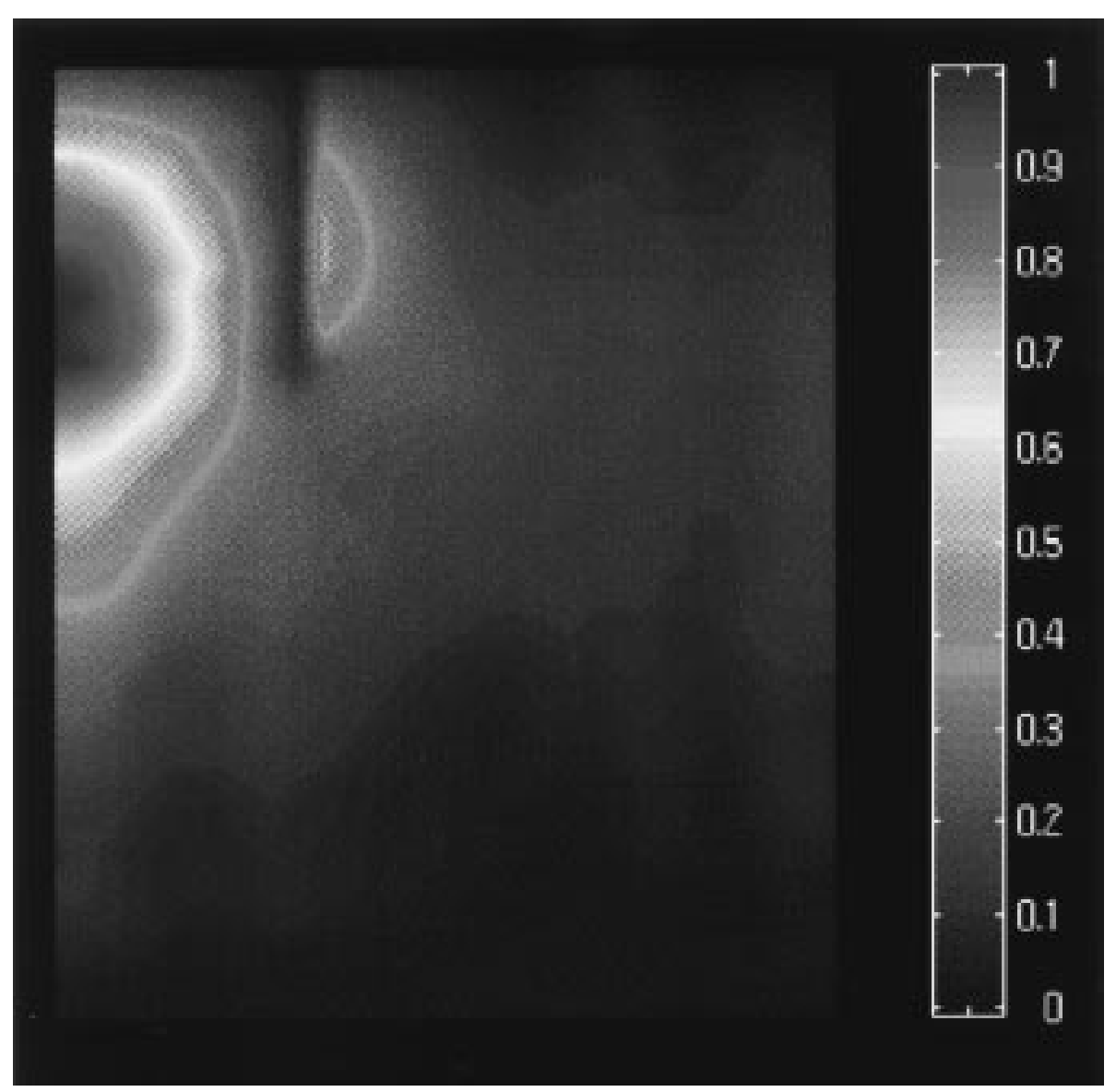

Figure 10. FDTD field pattern for 2D dielectric structure

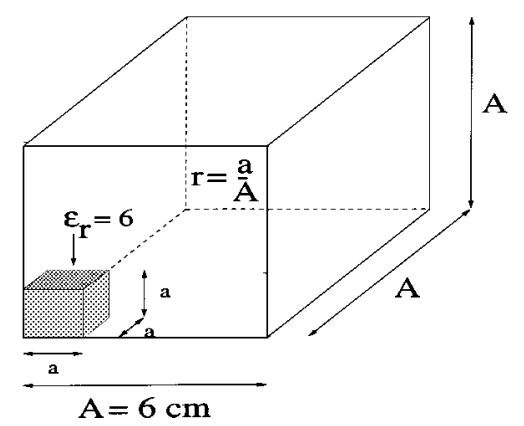

Figure 11. 3D resonator structure with varying dielectric in corner

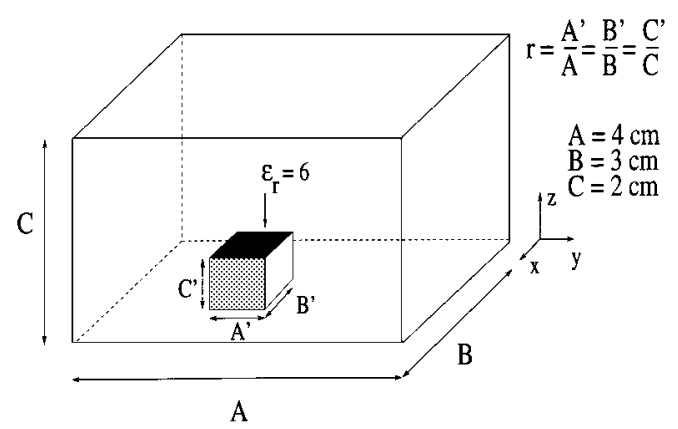

Figure 12. 3D resonator structure with varying dielectric in centre 
Table IV. Resonant frequencies for a resonator with a corner dielectric perturbation

\begin{tabular}{lccc}
\hline$r$ & FDTD $(40 \times 40 \times 40)$ & MRTD $(6 \times 6 \times 6)$ & MRTD $(8 \times 8 \times 8)$ \\
\hline 0.15 & 3.534 & 3.524 & 3.529 \\
0.25 & 3.500 & 3.489 & 3.497 \\
0.33 & 3.136 & 3.139 & 3.137 \\
\hline
\end{tabular}

Table V. Resonant frequencies for a resonator with a centre dielectric perturbation

\begin{tabular}{lllll}
\hline$r$ & IE/MoM, GHz & FEM & MRTD $(6 \times 6 \times 6)$ & MRTD $(8 \times 8 \times 8)$ \\
\hline $0 \cdot 10$ & $6 \cdot 220$ & $6 \cdot 1875$ & $6 \cdot 19$ & 6.20 \\
$0 \cdot 15$ & $6 \cdot 1535$ & $6 \cdot 1125$ & $6 \cdot 11$ & $6 \cdot 12$ \\
0.20 & $6 \cdot 02$ & $5 \cdot 9125$ & $5 \cdot 945$ & 5.975 \\
$0 \cdot 25$ & $5 \cdot 840$ & $5 \cdot 740$ & $5 \cdot 73$ & $5 \cdot 75$ \\
\hline
\end{tabular}

relative permittivity equal to 6 . In this case the varying dielectric is located in one corner of the structure, as shown in Figure 11. The S-MRTD simulations ran for 60,000 time steps, with $\Delta t=3 \cdot 0 \times 10^{-12} \mathrm{~s}$. FDTD simulations ran for 100,000 time steps with $\Delta t=2 \cdot 0 \times 10^{-12} \mathrm{~s}$. Note that FDTD needed to run for a high number of time steps in order to achieve convergence with the S-MRTD results. Results are shown in Table IV. Note that FDTD and S-MRTD have a high degree of convergence in the resonant frequency data.

The second 3D dielectric structure simulated is a varying dielectric cube with a relative permittivity equal to 6 . In this case the cube is located in the centre of the bottom face of the structure as shown in Figure 12. This structure has previously been simulated with tetrahedral FEM and an integral equation method $^{13}$ and is compared to S-MRTD in Table V. Once again a high degree of convergence is achieved between the methods.

\section{CONCLUSION}

MRTD schemes based on orthonormal wavelet expansions are derived and applied in the numerical analysis of simple resonant cavity structures. It is shown in this paper that a multiresolution expansion using Battle-Lemarie scaling functions has excellent correspondence with the FDTD method. In comparison with Yee's FDTD scheme, the examples suggest computer savings of two orders of magnitude with respect to the memory requirements.

\section{ACKNOWLEDGEMENTS}

This work has been made possible by a scholarship of the NATO science committee through the German Academic Exchange Service and by the U.S. Army Research Office.

\section{REFERENCES}

1. R. F. Harrington, Field Computation by Moment Methods, Krieger Publishing Company, Inc., Malabar, FL, 1982.

2. M. Krumpholz and P. Russer, 'Two-dimensional FDTD and TLM', Int. J. Numer. Model., 7 (2), $141-153$ (1993).

3. M. Krumpholz, C. Huber and P. Russer, 'A field theoretical comparison of FDTD and TLM', IEEE Trans. Microw. Theory Tech., 43 (8), 1935-1950 (1995).

4. S. G. Mallat, 'A theory for multiresolution signal decomposition: The wavelet representation', IEEE Trans. Pattern Anal. Mach. Intell., 11, 674-693 (1989).

5. B. Jawerth and W. Sweldens, 'An overview of wavelet based multiresolution analyses', SIAM Rev., 36 (3), $377-$ 412 (1994)

6. R. Robertson, E. Tentzeris, M. Krumpholz and L. P. B. Katehi, 'MRTD analysis of dielectric cavity structures', in 1996 IEEE MTT International Microwave Symposium Digest, pp. 1861-1864.

7. E. Tentzeris, M. Krumpholz and L. P. B. Katechi, 'Application of MRTD to printed transmission lines', in 1996 IEEE MTT International Microwave Symposium Digest, pp. 573-577.

8. M. Krumpholz, H. Winful and L. P. B. Katehi, 'Nonlinear time domain modelling by MRTD', in 1996 IEEE MTT International Microwave Symposium Digest, pp. 597-601.

9. G. Battle, 'A block spin construction of ondelettes', Commun. Math. Phys., 110, 601-615 (1987).

10. P. G. Lemarie, 'Ondelettes a localisation exponentielle', J. Math. Pures Appl., 67, pp. 227-236, 1988.

11. M. Krumpholz and L. P. B. Katehi, 'New prospects for time-domain analysis', IEEE Microw. Guid. Lett., 5, (11) , 382-384 (1995). 
12. M. Krumpholz and L. P. B. Katehi, 'MRTD: New time domain schemes based on multiresolution analysis', IEEE Trans. Microw. Theory Tech., 44, 555-571 (1995).

13. J. G. Yook and Kazem Sabet, Private communication.

\section{Authors' biographies:}

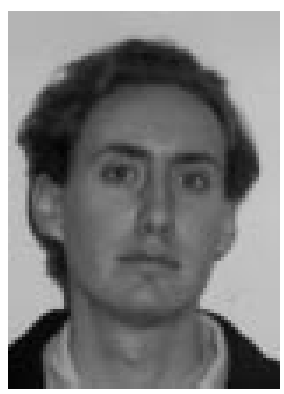

Rob Robertson was born in Fairfax, Virginia in 1971. He received a B.S.E.E. from the University of Wisconsin-Madison in 1995 and an M.S.E.E. from the University of Michigan in 1997. He is currently with the Radiation Laboratory at the University of Michigan as a Graduate Research Assistant, in pursuit of a $\mathrm{PhD}$. His research interests include the development of time-domain analysis techniques used in the analysis of antennas and wireless communication systems. Mr. Robertson is a member of I.E.E.E and Eta Kappa $\mathrm{Nu}$.

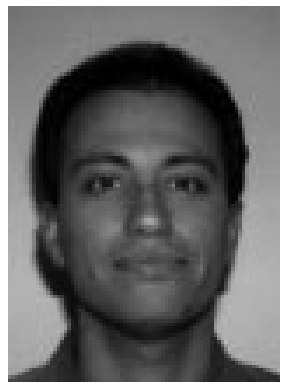

Emmanouil M. Tentzeris was born in Piraeus, Greece, in 1970. Mr. Tentzeris received his Electrical Engineering and Computer Science Diploma Degree (Suma Cum Laude) from the National Technical University of Athens, Greece in 1992 and the M.Sc. degree from the University of Michigan in 1993. Mr. Tentzeris is currently pursuing a Ph.D. degree at the University of Michigan. Since 1992, he has been a Graduate Research Assistant at the Radiation Laboratory, University of Michigan. His research interests include the development of novel numerical techniques and the application of the principles of Multiresolution Analysis for the simulation of microwave circuits used in wireless or satellite communication systems. He is the author and co-author of more than 20 papers in refereed journals and symposia proceedings. Mr. Tentzeris is a member of the I.E.E.E. and The Technical Chamber of Greece.

Michael Krumpholz was born in Bonn, Germany, in 1966. He received the Dipl.-Ing. degree and the Dr.Ing. degree in electrical engineering at the Technische University Aachen in 1991 and 1994. From 1991 to 1992, he was with the Lehrstuhl for Hochsfrequenztechnik, Technische University Munchen. In 1992, he joined the Ferdinand-Braun-Institut for Hochsfrequenztechnik in Berlin.

From 1994 to 1995, he was with the Radiation Laboratory at the University of Michigan in Ann Arbor. Since September 1995, he as been working with the Daimler-Benz Corporation.

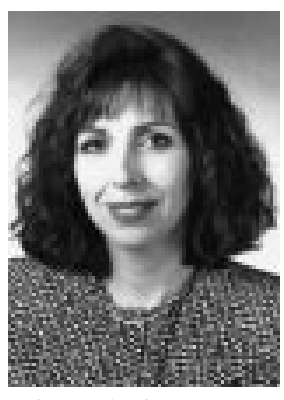

mixer designs.

She has been awarded with the IEEE AP-S W. P. King (Best Paper Award for Young Engineer) in 1984 the IEEE AP-S S. A. Schelkunoff Award (Best Paper Award) in 1985, the NSF Presidential Young Investigator Award and an URSI Young Scientist Fellowship in 1987, the Humboldt Research Award and The University of Michigan Faculty Recognition Award in 1994 and the IEEE MTT-S Microwave Prize in 1996. She is a Fellow of IEEE, and a member of IEEE AP-S, MTT-S, Sigma XI, Hybrid Microelectronics, URSI Commission $\mathrm{D}$ and a member of AP-S ADCOM from 1992 to 1995. Also, Prof. Katehi is an Associate Editor for the IEEE Transactions of the Microwave Theory and Techniques Society. 